\title{
ON HERMITIAN POLYNOMIAL OPTIMIZATION
}

\author{
MIHAI PUTINAR
}

\begin{abstract}
We compare three levels of algebraic certificates for evaluating the maximum modulus of a complex analytic polynomial, on a compact semi-algebraic set. They are obtained as translations of some recently discovered inequalities in operator theory. Although they can be stated in purely algebraic terms, the only known proofs for these decompositions have a transcendental character.
\end{abstract}

\section{INTRODUCTION}

Let $z=\left(z_{1}, \ldots, z_{d}\right)$ be the complex coordinates in $\mathbf{C}^{d}$. Then real coordinates of the underlying space $\mathbf{R}^{2 d}$ are denoted by $x=\left(x_{1}, \ldots, x_{2 d}\right)$, where $z_{k}=x_{k}+i x_{d+k}$. We will work in the polynomial algebra $\mathbf{R}[x]$, and consider there the convex hulls:

$$
\Sigma^{2}=\operatorname{co}\left\{p^{2} ; p \in \mathbf{R}[x]\right\}
$$

and

$$
\Sigma_{h}^{2}=\operatorname{co}\left\{|q|^{2} ; q \in \mathbf{C}[z]\right\} .
$$

It is easy to see that the cone of hermitian positive squares $\Sigma_{h}^{2}$ is a proper subset of $\Sigma^{2}$. Indeed, remark that

$$
\frac{\partial}{\partial z_{k}} \frac{\partial}{\partial \bar{z}_{k}}|q|^{2} \geq 0, \quad q \in \mathbf{C}[z]
$$

while the same Laplace operator has negative values on $\left(y_{k}-x_{k}^{2}\right)^{2}$. Note that both sets $\Sigma^{2}, \Sigma_{h}^{2}$ are multiplicatively closed and that $\Sigma^{2}$ is a module over $\Sigma_{h}^{2}$.

The theme of the present note is the following algebraic counterpart (sometimes called a certificate) for a polynomial optimization problem: let

$$
S=\left\{x \in \mathbf{R}^{2 d} ; p_{1}(x) \geq 0, \ldots, p_{m}(x) \geq 0\right\}
$$

be a compact basic semialgebraic set, and let $p$ be a positive polynomial on $S$. Under what conditions can one decompose $p$ as:

$$
p \in \Sigma_{h}^{2}+p_{1} \Sigma_{h}^{2}+\ldots+p_{m} \Sigma_{h}^{2},
$$

or

$$
p \in \Sigma^{2}+p_{1} \Sigma_{h}^{2}+\ldots+p_{m} \Sigma_{h}^{2},
$$

Mathematics Science Classification 2000: 47A12, 13J30, 46N10, 65K10.

Partially supported by the NSF grant DMS 0350911.

To appear in ARChIV DER MATHEMATIK. 
or

$$
p \in \Sigma^{2}+p_{1} \Sigma^{2}+\ldots+p_{m} \Sigma^{2} ?
$$

The latter decomposition (with respect to the so-called quadratic module respresenting the right hand term) is well understood today in purely algebraic terms, [18]. The middle one also holds under quite general assumptions, but the only available proof relies on the theory of subnormal tuples of operators (see [8, 20] and Section 4 below).

The first type decomposition is the strongest (with respect to a smaller set), and in general it requires additional bounds for the given polynomial $p$. It is our aim to present a series of cases (some of them of discovered only in the last couple of years) when (1) holds; they all require some functional analytic proofs and it would be interesting and desirable to have a unifying algebraic explanation for these phenomena. Decomposition (3) is currently used in optimization theory [17].

This note was completed while the author has benefited of excellent working conditions as a visitor of the University of Konstanz, Germany. He warmly thanks Alexander Prestel, Claus Scheiderer and their research group for hospitality.

\section{Preliminaries}

This section contains the main ingredient used to establish the correspondence between norm estimates of matrices and the structure of positive polynomials. Second, we collect here a few recent results about the (joint) numerical range and some related von Neumann type inequalities.

2.1. Cassier's lemma. Let $\mathbf{C}[z]_{n}$ and $\mathbf{R}[x]_{n}$ be the finite dimensional spaces of polynomials of degree less than or equal to $n$. Let $C=\Sigma_{h}^{2}+p_{1} \Sigma_{h}^{2}+\ldots+$ $p_{m} \Sigma_{h}^{2}$ be the convex cone associated to some real polynomials $p_{1}, \ldots, p_{m}$, and denote $C_{n}=C \cap \mathbf{R}[x]_{n}$. From the identity

$$
\left|z^{\alpha}+z^{\beta}\right|^{2}-\left|z^{\alpha}-z^{\beta}\right|^{2}=4 z^{\alpha} \bar{z}^{\beta},|\alpha|,|\beta| \leq n,
$$

we deduce $C_{2 n}-C_{2 n}=\mathbf{R}[x]_{2 n}$; hence the cone $C_{2 n}$ has interior points.

We can regard $C$ as the cone of positive elements in an order structure on the polynomial algebra. In analogy with the established algebraic terminology (see [18]] the cone $C$ will be called archimedian if $1 \in \operatorname{int} C_{2 n}$ for every (large enough) integer $n$. This is equivalent to asserting that for every element $f \in \mathbf{R}[x]_{2 n}$ there exists a large integer $N$ with the property $N-f \in C$.

For instance, assume either that there exists $M>0$ such that

$$
M-|x|^{2}=c_{1} p_{1}+\ldots+c_{m} p_{m},
$$

for some non-negative constants $c_{1}, \ldots, c_{m}$, or that there exists a positive constant $M$ such that, for every $k, 1 \leq k \leq d, M-x_{k}$ and $x_{k}+M$ both have such linear representations with non-negative coefficients. Then $C$ is archimedian, see [3] for a proof. 
The following technical but fundamental lemma was for the first time used by Cassier [3] in the derivation of some weighted sums of squares decompositions. Its essence and theoretical importance were recognized a long time before him by Eidelheit and Kakutani [10, 14] (we thank Markus Schweighofer for this information). The lemma was and will be used in conjunction with a Gelfand-Naimark-Segal construction and a proof by contradiction of the existence of the representation (1).

Lemma 2.1. Let $C=\Sigma_{h}^{2}+p_{1} \Sigma_{h}^{2}+\ldots+p_{m} \Sigma_{h}^{2}$ be an archimedian cone and assume that the polynomial $p \in \mathbf{R}[x]$ does not belong to $C$. Then there exists a linear functional $L: \mathbf{R}[x] \longrightarrow \mathbf{R}$, such that $L(1)>0$ and

$$
L(p) \leq 0 \leq L(c), c \in C .
$$

Proof. We include the proof for completeness, see [3] for the original. Start with a degree $2 n$ larger than that of $p$ and the assumption that $p$ does not belong to $C_{2 n}$. Since $\{p\}$ is disjoint of $\operatorname{int} C_{2 n}$, Minkowski's separation theorem implies the existence of a linear functional $L_{2 n}: \mathbf{R}[x]_{2 n} \longrightarrow \mathbf{R}$, such that

$$
L_{2 n}(p) \leq 0<L_{2 n}(c), c \in \operatorname{int} C_{2 n} .
$$

In particular $L_{2 n}(1)>0$ and $L_{2 n}$ is non-negative of $C_{2 n}$.

Next we extend $L_{2 n}$ to a functional $L_{2 n+2}: \mathbf{R}[x]_{2 n+2} \longrightarrow \mathbf{R}$ satisfying $L_{2 n+2}(p)=L_{2 n}(p)$ and being non-negative on $C_{2 n+2}$. This is done as follows: choose first, in view of Minkowski's theorem, a functional $L^{\prime}$ on $\mathbf{R}[x]_{2 n+2}$ separating ker $L_{2 n}$ from $\operatorname{int} C_{2 n+2}$, after checking that these convex sets are disjoint. Since $L^{\prime}(1)>0$, we can find a positive constant $\alpha$ such that $\alpha L^{\prime}(1)=L_{2 n}(1)$. Then $L_{2 n+2}=\alpha L^{\prime}$ will have the desired properties.

2.2. Numerical ranges. Let $T \in \mathcal{L}(H)$ be a linear bounded operator acting on a complex Hilbert space $H$. The numerical range of $T$ is the set

$$
W(T)=\{\langle T u, u\rangle ;\|u\|=1\} .
$$

By a classical theorem of Hausdorff this is a convex set, which contains in its closure the spectrum $\sigma(T)$ of $T$ and is contained in the disk centered at zero, of radius $\|T\|$. For instance, denoting by $J_{2}$ the Jordan cell of dimension 2 , one has $\sigma\left(J_{2}\right)=\{0\}$, wile $W\left(J_{2}\right)=\{z ;|z| \leq 1 / 2\}$ and $\left\|J_{2}\right\|=1$. In general, the numerical range is easier to estimate than the spectrum, see for instance [25].

Let $U$ be an open neighborhood of the spectrum $\sigma(T)$ and let $F$ be a complex analytic function defined on $U$. Then one can define a multiplicative functional calculus, after Riesz and Dunford:

$$
f(T)=\int_{\gamma}(z-T)^{-1} \frac{d z}{2 \pi i},
$$

where $\gamma$ is a system of rectifiable curves surrounding $\sigma(T)$ inside $U$. The continuity of this calculus is expressed as:

$$
\|f(T)\| \leq C_{\gamma, T}\|f\|_{\infty, \gamma}
$$


where the positive constant $C_{\gamma, T}$ increases in general to infinity as soon as $\gamma$ approaches the spectrum, see for instance [24]

A remarkable inequality, stronger in general than the preceding one, asserts:

$$
\|p(T)\| \leq\|p\|_{\infty, \mathbf{r} D}, \quad p \in \mathbf{C}[z],
$$

where $r=\|T\|$ and $\mathbf{D}$ stands for the open unit disk. This estimate is due to von Neumann and it was the source of a rich development in functional analysis, see $[24,25]$. Note that in von-Neumann's inequality the spectrum of $T$ can touch the boundary of the disk $r \mathbf{D}$ on which one maximizes the function $|p|$.

It turns out that a von-Neumann type inequality holds for every linear operator $T$, with a uniform bound on the closed numerical range. To be more specific,

$$
\|p(T)\| \leq C_{W(T)}\|p\|_{\infty, W(T)}, p \in \mathbf{C}[z] .
$$

This was recently proved in [7]. The universal constant $C_{W(T)}$ has a geometric character, revealed a long time ago by studies studies in potential theory [22]. We will call it the (Carl) Neumann constant of the convex set. For examples the reader can consult [6,22].

The idea behind the proof of (6) is the following. The convex set $W(T)$ has a rectifiable boundary, along which the form $\Re \frac{d z}{2 \pi i(z-w)}$ is non-negative for every inner point $w \in W(T)$. For a polynomial $p \in \mathbf{C}[z]$ one can define its "double layer" transform:

$$
\tilde{p}(w)=\int_{\partial W(T)} p(z) \Re \frac{d z}{2 \pi i(z-w)} .
$$

One remarks, using elementary function theory, that the transform $p \mapsto \tilde{p}$ extends continuously and bijectively to the algebra of continuous functions on $\partial W(T)$. Finally, one replaces $w$ by $T$, via a standard limiting process, and one obtains:

$$
\tilde{p}(T)=\int_{\partial W(T)} p(z) E_{T}(d z),
$$

with a positive, operator valued measure $E_{T}(d z)=\lim _{\rho \rightarrow 1} \Re \frac{d z}{2 \pi i}(z-\rho T)^{-1}$, (where we assume $0 \in W(T)$ and $\rho<1$ ). Then we infer

$$
\|\tilde{p}(T)\| \leq\|p\|_{\infty, W(T)} \leq C_{W(T)}\|\tilde{p}\|_{\infty, W(T)} .
$$

See [22] for details.

In the next section we will translate these inequalities into algebraic form.

2.3. The Fantappiè transform. When passing from one to several complex variables, von Neumann type inequalities become more involved and more interesting. For instance, we have Ando's theorem asserting that

$$
\left\|p\left(T_{1}, T_{2}\right)\right\| \leq\|p\|_{\infty, \mathbf{D}^{2}}, p \in \mathbf{C}\left[z_{1}, z_{2}\right],
$$


whenever $T_{1}, T_{2} \in \mathcal{L}(H)$ are commuting, contractive $\left(\left\|T_{1}\right\|,\left\|T_{2}\right\| \leq 1\right)$ linear operators. It is however surprising that this inequality is not valid in three or more variables, cf. $[1,12,25]$.

Or consider a spherical contractive tuple $T=\left(T_{1}, \ldots, T_{d}\right)$ of commuting operators: $T_{1}^{*} T_{1}+\ldots+T_{d}^{*} T_{d} \leq I$. Due to the pioneering work of Drury [9] we know today that there exists an archetypal tuple in this class: the multiplication operators by the complex variables $M=\left(M_{z_{1}}, \ldots, M_{z_{d}}\right)$ on the Hilbert space of analytic functions in the unit ball $\mathbf{B}_{d} \subset \mathbf{C}^{d}$, with reproducing kernel $(1-z \cdot \bar{w})^{-1}$. In this case, for instance when $d=2$,

$$
\lim _{n \rightarrow \infty}\left\|\left(2 M_{z_{1}} M_{z_{2}}\right)^{n}\right\|=\infty
$$

although $\left\|2 z_{1} z_{2}\right\|_{\infty, \mathbf{B}_{2}}=1$. Thus there is no hope to have an inequality of the type (6), with a universal constant, on the unit ball of $\mathbf{C}^{d}, d>1$. This example will be resumed at the end of the note.

On the other hand there is a way to obtain a crude estimate for $p(T)$ when $T$ is a spherical contraction. Namely, by repeating the double layer technique used in proving the single variable estimate on the numerical range of an operator, one can introduce the Fantappiè transform

$$
\tilde{p}(z)=\int_{\partial \mathbf{B}_{d}} \frac{p(w) d \sigma(w)}{1-z \cdot \bar{w}},
$$

of a polynomial $p$, where $d \sigma$ denotes the normalized surface measure on the sphere. This leads to an integral representation, valid for every commutative $d$-tuple $T$ fulfilling the joint numerical range condition

$$
\left|\left\langle T_{1} u, u\right\rangle\right|^{2}+\ldots+\left|\left\langle T_{d} u, u\right\rangle\right|^{2} \leq 1,\|u\| \leq 1 .
$$

We will write in short then, in analogy with the single operator situation, $W(T) \subset \overline{\mathbf{B}_{d}}$. Specifically,

$$
\frac{1}{2} \tilde{p}(T)+\frac{1}{2} p(0)=\int_{\partial \mathbf{B}_{d}} p(w) E_{T}(d w)
$$

where $E_{T}(d w)=\lim _{\rho \rightarrow 1} \Re\left[d \sigma(w)(1-\rho T \cdot \bar{w})^{-1}\right]$. All together, these computations yield the following estimate:

$$
\|p(T)\| \leq\|\Gamma p\|_{\infty, \partial \mathbf{B}_{d}}, p \in \mathbf{C}[z], W(T) \subset \overline{\mathbf{B}_{d}},
$$

where the operator $\Gamma$ is diagonal on the homogeneous decomposition $p=$ $p_{0}+p_{1}+\ldots+p_{n}$ :

$$
\Gamma p=p_{0}+2 \frac{d !}{1 !} p_{1}+\ldots+2 \frac{(d+n-1) !}{n !} p_{n} .
$$

Details can be found in [16]. 


\section{MAIn RESUltS}

We turn now to hermitian forms decompositions of the type (1), with the direct aim at translating into such terms some of the estimates discussed in the preliminaries. All algebraic decompositions below are similar, although the nature of the estimates they are based on is different. More important, however, the pattern of deducing them is the same.

3.1. One complex variable. Note that the definition of the archimedian cone $\Sigma_{h}^{2}+p_{1} \Sigma_{h}^{2}+\ldots+p_{m} \Sigma_{h}^{2}$ is dependent on the choice of the defining polynomials $p_{1}, \ldots, p_{m}$. Without changing the compact supporting set $S=$ $\left\{x ; p_{j}(x) \geq 0,1 \leq j \leq m\right\}$ one can add to the system $p_{m+1}(x)=R^{2}-\|x\|^{2}$, with large $R$, so that $S$ is unchanged and the new cone is archimedian.

Theorem 3.1. Let $S=\left\{x \in \mathbf{R}^{2} ; l_{j}(x) \geq 0,1 \leq j \leq m\right\}$ be a convex compact polygon defined by the real affine functions $l_{j}$ and let $C_{S}$ be Neumann's constant associated to $S$. Then for every $R>C_{S}$ and polynomial $p \in \mathbf{C}[z]$ one has:

$$
R^{2}\|p\|_{\infty, S}^{2}-|p(z)|^{2} \in \Sigma_{h}^{2}+l_{1} \Sigma_{h}^{2}+\ldots+l_{m} \Sigma_{h}^{2} .
$$

Proof. First we will check that the associated cone of hermitian polynomials is archimedian. Denote $l_{j}(x)=a_{j} . x-b_{j}$, with $a_{j} \in \mathbf{R}^{2}$ and $b_{j} \in \mathbf{R}$. Since the set $S$ defined by the inequalities $a_{j} . x \leq b_{j}$ is compact, the convex cone generated by the vectors $a_{j}$ is the whole space. Otherwise, the polar would be non-empty, hence there would exist a vector $v$ such that $t v \in S$ for all $t>0$. Therefore for every every coordinate $x_{k}, k=1,2$, there exists a positive constant $R_{k}$ such that

$$
R_{k}-x_{k}=\sum_{j} c_{j} l_{j}+c_{0}, \quad x_{k}+R_{k}=\sum d_{j} l_{j}+d_{0},
$$

with non-negative coefficients $c_{0}, c_{1}, \ldots, d_{0}, \ldots, d_{m}$. This shows that the cone $\Sigma_{h}^{2}+l_{1} \Sigma_{h}^{2}+\ldots+l_{m} \Sigma_{h}^{2}$ is archimedian.

Assume by contradiction that $q(z, \bar{z})=M^{2}\|p\|_{\infty, S}^{2}-|p(z)|^{2}$ does not belong to the right hand side cone $C$. According to Cassier's lemma, one can find a linear functional $L$, with the separation property $L(q) \leq 0 \leq$ $L(c), c \in C$. In addition $L(1)>0$. The polynomial algebra $\mathbf{C}[z]$ is then endowed with a positive semidefinite sesquilinear form

$$
\langle f, g\rangle=L(f \bar{g}) .
$$

Let $H$ denote the associated Hilbert space, i.e. the completion of the quotient $\mathbf{C}[z] /\{h ; L(h \bar{h})=0\}$. Since the cone $C$ is archimedian, the multiplication operator $M_{z}$ by the complex variable is bounded with respect to $L$, hence it extends and remains bounded on $H$ :

$$
R^{2}\|h\|^{2}-\left\|M_{z} h\right\|^{2}=L\left(\left(R^{2}-|z|^{2}\right) h \bar{h}\right) \geq 0, h \in \mathbf{C}[z] .
$$

Moreover, the numerical range of $M_{z}$ is contained in $S$, that is

$$
\left\langle M_{z} h, h\right\rangle \in S,\|h\|=1 .
$$


Indeed, writing the defining functions in complex form $l_{j}(x)=\Re\left(\alpha_{j} z\right)-b_{j}$ one finds, for a unit vector $h$,

$$
\Re\left[\alpha_{j}\left\langle M_{z} h, h\right\rangle\right]-b_{j}=\left\langle\left(\Re\left(\alpha_{j} M_{z}\right)-b_{j}\right) h, h\right\rangle=L\left(l_{j}|h|^{2}\right) \geq 0 .
$$

To finish the proof we invoke inequality (6). It applies to the operator $M_{z}$ and gives $\left\|p\left(M_{z}\right)\right\| \leq C_{s}\|p\|_{\infty, S}$, and in particular

$$
L\left(|p|^{2}\right)=\langle p, p\rangle=\left\langle p\left(M_{z}\right) 1, p\left(M_{z}\right) 1\right\rangle \leq\left[C_{s}\|p\|_{\infty, S}\right]^{2} L(1) .
$$

On the other hand, $L(1)>0$,

$$
L\left(R^{2}-|p|^{2}\right) \leq 0
$$

and the constant $R$ was supposed bigger than $C_{S}\|p\|_{\infty, S}$, a contradiction.

A couple of remarks are in order. First what happens if one works with an archimedian cone $C=\Sigma_{h}^{2}+p_{1} \Sigma_{h}^{2}+\ldots+p_{m} \Sigma_{h}^{2}$ defined by higher degree polynomials? Then the above statement holds true, with

$$
R>\sup _{\Re p_{j}(T) \geq 0}\|p(T)\|,
$$

where the supremum is taken among all linear operators $T$ acting on a Hilbert space. They are automatically bounded since, by Archimedes axiom, $\rho^{2}-|z|^{2} \in C$, for some large $\rho$. Then one can invoke von Neumann's inequality:

$$
\sup _{\Re p_{j}(T) \geq 0}\|p(T)\| \leq \sup _{T^{*} T \leq \rho^{2}}\|p(T)\| \leq\|p\|_{\infty, \rho \mathbf{D}}
$$

However, in general there is very little information about the supremum in (10). This would requires functoriality transforms for the numerical range of a linear operator, and there are very few positive results in this direction, see $[22]$ and the references cited there.

Second, what happens if a compact set is defined by a non-archimedian cone? For instance, the elements of

$$
C=\Sigma_{h}^{2}-|z|^{4} \Sigma_{h}^{2}
$$

are simultaneously non-negative only at $z=0$. Let $J_{2}$ be the Jordan block of dimension 2. Then $t J_{2}$ has norm $t$, for every $t>0$, in spite of the fact that $p\left(t J_{2}, t J_{2}^{*}\right) \geq 0$ for all $p \in C$. Choosing by convention to put all adjoins in the monomials of $p$ to the left of the powers of $t J_{2}$. Algebraically, this amounts to the fact that there exists no universal constant $R$, so that

$$
R^{2}\|z\|_{\infty,\{0\}}^{2}-|z|^{2} \in \Sigma_{h}^{2}-|z|^{4} \Sigma_{h}^{2}
$$

Indeed, this would mean

$$
-|z|^{2}=\sum\left|f_{j}(z)\right|^{2}-|z|^{4} \sum\left|g_{k}(z)\right|^{2},
$$

with $f_{j}, g_{k}$ all complex analytic. But then $f_{j}(0)=0$ for all $j$, hence one can divide by $z$ and find:

$$
-1=\sum\left|f_{j}^{\prime}(0)\right|^{2}
$$


a contradiction.

3.2. The ball and polydisk. In general, in the case of several complex variables, the weighted hermitian sums of squares cannot detect the largest value of the modulus of a complex polynomial. To find the sharp bounds which still yield such a decomposition requires a rather sophisticated functional analytic machinery, see for instance [1]. If we are seeking merely some (crude) estimates in this direction, then the Fantappiè transform technique can help. We discuss below only two applications.

Theorem 3.2. Let $p=p_{0}+\ldots+p_{n} \in \mathbf{C}[z], z \in \mathbf{C}^{d}$, be a polynomial decomposed into its homogeneous parts. If

$$
R>\left\|p_{0}+2 \frac{d !}{1 !} p_{1}+\ldots+2 \frac{(d+n-1) !}{n !} p_{n}\right\|_{\infty, \mathbf{B}_{n}},
$$

then

$$
R^{2}-|p(z)|^{2} \in \Sigma_{h}^{2}+\left(1-\|z\|^{2}\right) \Sigma_{h}^{2} .
$$

Proof. The proof can be modeled after that of Theorem 3.1: arguing by contradiction, we separate the left hand side polynomial from the (obviously archimedian) hermitian cone by the functional $L$. The tuple $M$ of multiplication operators with the complex coordinates has then the joint numerical range contained in the closed unit ball. Indeed, for a unit vector $h \in \mathbf{C}[z], L\left(|h|^{2}\right)=1$, Cauchy-Schwarz inequality gives:

$$
\sum_{j}\left|L\left(M_{z_{j}}|h|^{2}\right)\right|^{2} \leq \sum_{j} L\left(\left|M_{z_{j}} h\right|^{2}\right)=L\left(\|z\|^{2}|h|^{2}\right) \leq L\left(|h|^{2}\right)=1 .
$$

Then one invokes inquality (8).

A similar proof can be adapted to the case of the polydisk. We use a proper normalization and work on $\Delta=\prod_{1}^{d} \frac{1}{\sqrt{d}} \mathbf{D}$, so that the distinguished boundary of $\Delta$ lies on the unit sphere. The dual (polar) domain is $\Delta_{*}=$ $\left\{w \in \mathbf{C}^{d} ; \sum_{j}\left|w_{j}\right| \leq \sqrt{d}\right\}$. It is a Reinhardt domain, and the transform

$$
\tilde{p}(z)=\int_{\Delta_{*}} \frac{p(w) d \omega(w)}{1-z \cdot \bar{w}},
$$

is still diagonalized by the monomials. Above $\omega$ is the unit volume measure on $\Delta_{*}$. Then $\int_{\Delta_{*}} p(w) d \omega(w)=p(0)$ and, as before, the map $\Gamma(\tilde{p}+p(0))=$ $2 p$ is invertible, see [16] for details. With these conventions we have the following result.

Proposition 3.3. For a polynomial $p \in \mathbf{C}[z]$ and constant $R>\|\Gamma p\|_{\infty, \Delta_{*}}$ the decomposition

$$
R^{2}-|p(z)|^{2}=\Sigma_{h}^{2}+\left(1-d\left|z_{1}\right|^{2}\right) \Sigma_{h}^{2}+\ldots+\left(1-d\left|z_{d}\right|^{2}\right) \Sigma_{h}^{2},
$$

holds. 
The case $d=2$ stands aside, due to Ando's theorem. We state it in a form comparable to the other decompositions discussed in this note. The proof is very similar to that of Theorem 3.1 and will be omitted.

Proposition 3.4. In dimension $d=2$, let $p \in \mathbf{C}[z]$ and $R>\|p\|_{\infty, \mathbf{D}^{2}}$. Then

$$
R^{2}-|p(z)|^{2}=\Sigma_{h}^{2}+\left(1-\left|z_{1}\right|^{2}\right) \Sigma_{h}^{2}+\left(1-\left|z_{2}\right|^{2}\right) \Sigma_{h}^{2} .
$$

This remarkable fact, well noted and exploited in modern operator theory, has no known algebraic proof, see also the article [5].

\section{Subnormal tuples}

So far we were concerned with a weighted sum of hermitian squares. For the sake of completeness we reproduce below a known result for the mixed decomposition (type (2) in the introduction).

Theorem 4.1. Let $S=\left\{z \in \mathbf{C}^{d} ;\left|p_{j}(z)\right| \leq 1,1 \leq j \leq m\right\}$ be a compact semialgebraic set, where $p_{j}$ are complex polynomials. Assume that the associated hermitian cone $C=\Sigma^{2}+\left(1-\left|p_{j}\right|^{2}\right) \Sigma_{h}^{2}+\ldots+\left(1-\left|p_{m}\right|^{2}\right) \Sigma_{h}^{2}$ is archimedian. Then for every real polynomial $p \in \mathbf{R}[x]$ which is strictly positive on $S$ we have $p \in C$.

Proof. We merely sketch the main steps in the proof. For details see [21]. As before, in view of Cassier's lemma, we separate $p$ from the cone $C$ by a functional $L$. And define the Hilbert space, separated completion $H$ of $\mathbf{C}[z]$. The tuple $M$ of multipliers by the complex coordinates is commutative and bounded on $H$.

The fact that $L\left(h^{2}\right) \geq 0$ for every real polynomial $h$ implies that $M$ is a subnormal tuple. That is there exists a larger Hilbert space $H \subset K$ and a commutative normal tuple $N$, acting there, such that $\left.N\right|_{H}=M$. Moreover, one knows that, choosing $N$ minimal will assure that the joint spectra satisfy the inclusion $\sigma(N) \subset \sigma(M)$.

On the other hand, the spectral mapping theorem for the joint spectrum implies $\sigma(M) \subset S$. Therefore, putting all adjoins $M^{*}$ to the left of the powers of $M$, we get

$$
0 \geq L(p)=\left\langle p\left(M, M^{*}\right) 1,1\right\rangle=\left\langle p\left(N, N^{*}\right) 1,1\right\rangle=\int_{S} p(x)\left\langle E_{N}(d x) 1,1\right\rangle,
$$

where $E_{N}$ denotes the spectral measure of the normal system $N$. But this is a contradiction because $\left\langle E_{N}(d x) 1,1\right\rangle$ is a non-trivial positive measure and the polynomial $p$ was supposed to be strictly positive on $S$.

Again, an algebraic proof and explanation of Theorem 4.1 are missing.

Most of the results discussed above also hold for pluriharmonic polynomails $\Re p(z), p \in \mathbf{C}[z]$. This is due to the fact that the semi-spectral measure measure $E_{T}$ appearing in the Fanttapiè or double layer potential transforms is non-negative. We refer to $[16,22]$ for details. 
Example. To finish we go back for an example to Drury [9] and look at the polynomial $2 z_{1} z_{2}$ on the unit ball of $\mathbf{C}^{2}$. As mentioned before, the norm of high powers of this monomial, on the Hilbert space with reproducing kernel $(1-z \cdot \bar{w})^{-1}$, can be bounded from below (using Stirling's formula):

$$
\left\|2^{n}\left(z_{1} z_{2}\right)^{n}\right\|^{2} \geq 2^{2 n} \frac{n !^{2}}{(2 n) !} \approx \frac{\pi}{2} \sqrt{n} .
$$

On the other hand, the Fantappiè transform method gives, for any commuting tuple $T$ with the joint numerical range in the unit ball:

$$
\left\|2^{n}\left(T_{1} T_{2}\right)^{n}\right\| \leq 2 \frac{(2 n+1) !}{(2 n) !}\left\|2^{n}\left(z_{1} z_{2}\right)^{n}\right\|_{\infty, \mathbf{B}_{2}}=4 n+2 .
$$

Thus, for $R^{2} \leq \frac{\pi}{2} \sqrt{n}$, the polynomial $R^{2}-4^{n}\left(\left|z_{1} z_{2}\right|\right)^{2 n}$ does not belong to $\Sigma_{h}^{2}+\left(1-\|z\|^{2}\right) \Sigma_{h}^{2}$, while for $R^{2}>(4 n+2)^{2}$ it does. On the other hand, in view of Theorem 4.1,

$$
\rho^{2}-4^{n}\left(\left|z_{1} z_{2}\right|\right)^{2 n} \in \Sigma^{2}+\left(1-\|z\|^{2}\right) \Sigma_{h}^{2}
$$

whenever $\rho>1$.

\section{Final REMARKS}

The results exposed above reveal a sensible difference between optimizing the modulus of a complex polynomial with respect to symmetric, hermitian or mixed weighted sums of squares, cf. decompositions (1)-(3). The last decade has known a lot of activity, oriented towards theoretical or very practical aims, around positivity certificates based on real symmetric forms of the form $(3)$, see $[17,19]$.

On the other hand hermitian forms, and complex Hilbert space methods, form the skeleton for most of the modern functional analysis. And beyond, to cite for instance the classical polynomial root separation criteria of Schur and Takagi, see [15].

The sums of squares structure of positive hermitian polynomials goes back at least to Quillen's 1968 article [23]. On a more recent date such structures have recurrently appeared in the geometric aspects of the theory of functions of several complex variables, see for instance [4].

Not unrelated to the topics of the present note, and the operator theory background, are the recent studies of sums of squares in a free ${ }^{*}$-algebra [13].

\section{REFERENCES}

[1] J. AGLER, J.E. McCARTHY, Pick interpolation and Hilbert function spaces. Graduate Studies in Mathematics. 44. Providence, RI: American Mathematical Society (2002).

[2] J.A., BALL, T.T. TRENT, Unitary colligations, reproducing kernel Hilbert spaces, and Nevanlinna-Pick interpolation in several variables. J. Funct. Anal. 157, No.1, 1-61 (1998). 
[3] G. CASSIER, Cassier, Problme des moments sur un compact de $\mathbf{R}^{n}$ et dcomposition de polynmes a plusieurs variables. J. Funct. Anal. 58, 254-266 (1984).

[4] D.W. CATLIN, J.P. D'ANGELO, An isometric imbedding theorem for holomorphic bundles. Math. Res. Lett. 6, No.1, 43-60 (1999).

[5] J.B. COLE, J. WERMER, Ando's theorem and sums of squares. Indiana Univ. Math. J. 48, No.3, 767-791 (1999).

[6] M. CROUZEIX, B. DELYON, Some estimates for analytic functions of strip or sectorial operators. Arch. Math. 81, No.5, 559-566 (2003).

[7] B. DELYON, F. DELYON, Generalization of von Neumann's spectral sets and integral representation of operators. Bull. Soc. Math. Fr. 127, No.1, 25-41 (1999).

[8] O.DEMANZE, Problème des moments multi-dimentionnel et sous-normalité jointe. Thèse de doctorat. Univ. Lille I, 2002.

[9] S.W. DRURY, A generalization of von Neumann's inequality to the complex ball. Proc. Am. Math. Soc. 68, 300-304 (1978).

[10] M. EIDELHEIT, Zur Theorie der konvexen Mengen in linearen normierten Räumen. Studia Math. 6, 104-111 (1936).

[11] J. ESCHMEIER, M. PUTINAR, Spherical contractions and interpolation problems on the unit ball. J. Reine Angew. Math. 542, 219-236 (2002).

[12] C. FOIAS, A.E. FRAZHO, The commutant lifting approach to interpolation problems. Operator Theory: Advances and Applications, 44. Basel. Birkhäuser, (1990).

[13] J.W.HELTON, S. McCULLOUGH, M. PUTINAR, Non-negative hereditary polynomials in a free *-algebra. Math. Zeitschrift 250, 515-522 (2005)

[14] S. KAKUTANI, Ein Beweis des Satzes von M. Eidelheit über konvexe Mengen. Proc. Imp. Acad. Tokyo 13, 93-94 (1937).

[15] M.G. KREIN, M.A. NAIMARK, The method of symmetric and Hermitian forms in the theory of separation of the roots of algebraic equations. (Translated from the Russian by O. Boshko and J. L. Howland). Linear Multilinear Algebra 10, 265-308 (1981).

[16] J.E.McCARTHY, M. PUTINAR, Positivity aspects of the Fantappiè transform. J. d'Analyse Math., to appear.

[17] J.B. LASSERRE, Global optimization with polynomials and the problem of moments. SIAM J. Optim. 11, No.3, 796-817 (2001).

[18] A. PRESTEL, C.N. DELZELL, Positive polynomials. From Hilbert's 17th problem to real algebra. Springer Monographs in Mathematics. Berlin: Springer, (2001).

[19] A. PRESTEL, Representation of real commutative rings. Expo. Math. 23, 89-98 (2005).

[20] M. PUTINAR, Sur la complexification du problme des moments. C. R. Acad. Sci., Paris, Sr. I 314, No.10, 743-745 (1992).

[21] M.PUTINAR, Positive polynomials on compact semi-algebraic sets. Indiana Univ. Math. J. 42, No.3, 969-984 (1993).

[22] M. PUTINAR, S. SANDBERG, A skew normal dilation on the numerical range of an operator. Math. Ann. 331, No.2, 345-357 (2005).

[23] D.G. QUILLEN, On the representation of hermitian forms as sums of squares. Invent. Math. 5, 237-242 (1968).

[24] F. RIESZ, B. SZ.-NAGY, Functional analysis. Transl. from the 2nd French ed. by Leo F. Boron. Reprint of the 1955 orig. publ. by Ungar Publ. Co., Dover Books on Advanced Mathematics. New York: Dover Publications, Inc., (1990).

[25] B. SZ.-NAGY, C. FOIAS, Analyse harmonique des opérateurs de l'espace de Hilbert. Budapest: Akademiai Kiado; Paris: Masson et Cie, 1967. 
Department of Mathematics, University of California, Santa Barbara, CA 93106, U.S.A.

E-mail address: mputinar@math.ucsb.edu 